\author{
Jonathan D. Blotter \\ Robert L. West \\ Mechanical Engineering Dept. \\ Virginia Tech \\ Blacksburg, VA 24061-0238
}

\title{
Experimental and Analytical Power Flow in Beams Using a Scanning Laser Doppler Vibrometer
}

\begin{abstract}
An experimental spatial power-flow (ESPF) method is presented. This method provides a spatially continuous model of the power-flow vector field derived from experimental measurements. The power-flow vector field clearly indicates locations of energy sources and sinks as well as paths of energy transmission. In the ESPF approach, a scanning laser Doppler vibrometer acquires spatially dense measurements of the vibrating test structure. These measurements are used in solving for a spatially continuous 3-dimensional complex-valued model of the steady-state dynamic response. From this experimentally derived dynamics model, a spatial representation of the power flow is computed. (c) 1996 John Wiley \& Sons, Inc.
\end{abstract}

\section{INTRODUCTION}

Analytical and experimental methods that seek to develop a spatial map of the power or energy flow in vibrating structures are generally referred to as power-flow methods. The concept behind these methods is that energy sources and sinks as well as paths of energy transmission in a structure can be identified. Once the energy path has been determined, the structure can be designed or controlled to dissipate and channel the energy as required. The design or control problem becomes a problem of "managing" the energy in the system. The interest in power flow is derived from the promise for design and control, given the ability to identify the magnitude and location of energy sources and sinks. As a result, powerflow techniques have been proposed and applied to a broad range of problems associated with structural dynamics, such as noise control, structural acoustics, dynamic characterization, and nondestructive evaluation.

Recently, many analytical power-flow solutions have been developed that provide a spatial representation of the dynamic response (Hambric, 1990a; Bouthier and Bernhard, 1992). However, in these analytical models many underlying assumptions on the material properties, boundary conditions, and external loadings must be made to obtain results. Several experimental techniques that involve the use of accelerometers and other structurally mounted measuring devices have also been developed (Pavic, 1976; Palmer et al., 1993). This type of experimental approach does not provide a spatial representation of the system dynamics and often neglects the near-field effects encountered at the boundaries and the locations of the external forces. Therefore, a power-flow

Received November 23, 1994; Accepted December 21, 1995.

Shock and Vibration, Vol. 3, No. 5, pp. 325-336 (1996)

(C) 1996 by John Wiley \& Sons, Inc.

CCC $1070-9622 / 96 / 050325-12$ 
technique that provides a spatially continuous representation of the power flow and also incorporates the true boundary conditions is desired.

This article presents an experimental spatial power flow (ESPF) method that satisfies these requirements. The ESPF uses a scanning laser Doppler vibrometer (SLDV) to provide spatially dense measurements of the structure's dynamic response. These experimental measurements of the response inherently include the boundary conditions and material properties of the structure. The 2-dimensional (2-D) raw laser measurements are used to solve for the continuous complex-valued 3-dimensional (3-D) velocity field. This velocity field can be integrated in time to obtain a 3-D representation of the displacement field. In this article, Bernoulli-Euler beam theory is used to express the generalized forces in terms of the displacements. Although Bernoulli-Euler beam theory is used, any mechanics model that relates the generalized forces to the displacements could be incorporated. The power flow is then computed by taking the dot product of the generalized forces and velocities. This powerflow technique is a novel approach in that it provides a spatially continuous representation of the power flow in the structure that is based on actual measurements of the system response.

In this study the derivation of the power-flow equations for a beam are briefly reviewed. The underlying assumptions of the analytical approach are discussed and quantified. The ESPF method is then presented and discussed in detail. The ESPF method is illustrated by showing results for each step of the procedure. Finally, a comparison of simulated experimental and analytical results is presented. The importance of accurate estimates of the material properties and the boundary conditions is manifested in the results, and the ability to extract these values from experimental power flow is made evident.

\begin{abstract}
ANALYTICAL APPROACH
An overview of the derivation of the power-flow equations for an experimental beam system that approximates free-free end conditions is presented in this section. This overview provides significant insight into the parameters that effect the power flow. This derivation is followed by a discussion of the underlying assumptions that effect analytical power-flow results. The effects of the assumptions dealing with the material properties, boundary conditions, and near-field effects are discussed. The focus throughout this article is on beams, with the understanding that similar assumptions apply to rods, plates, and shells.
\end{abstract}

\section{Theoretical Development}

In this section, Hamilton's extended principle is used to derive the equation of motion and the boundary conditions for a beam suspended from a bungee cord. The experimental system and the analytical model are shown in Fig. 1. As shown, the suspension system is modeled by a transverse spring and damper in parallel. The experimental system shows a foam pad at the opposite end of

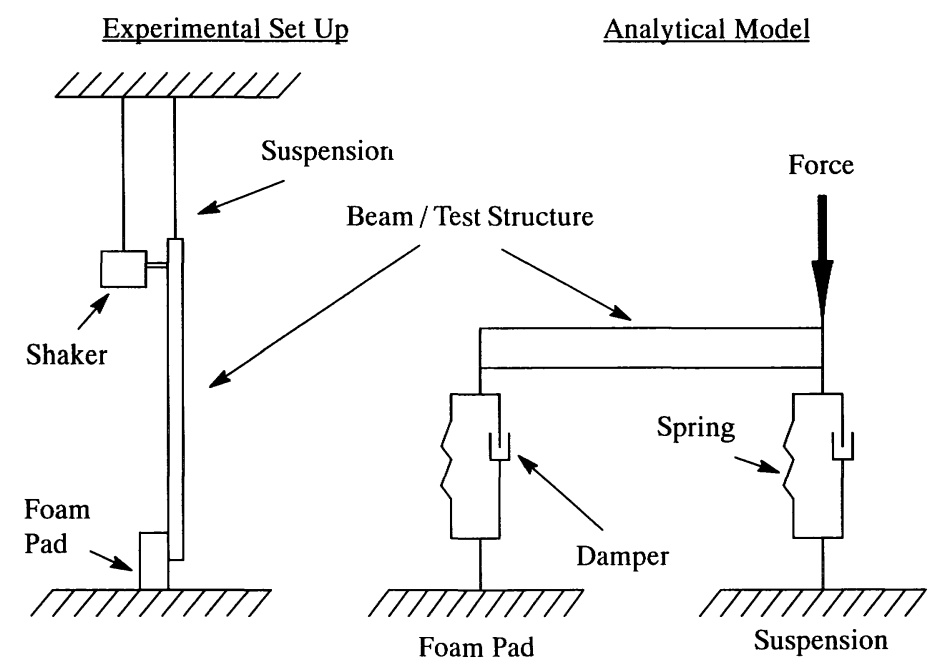

FIGURE 1 Experimental setup and analytical model. 
the suspension. This foam pad is used to prevent any rigid body rotation or swaying of the beam during the test. The foam pad is also modeled with a transverse spring and damper in parallel.

The analytical model used throughout this study is based on the Bernoulli-Euler beam formulation, which neglects the effects of rotatory inertia and shear deformation. The use of the Bernoulli-Euler model can be justified for beams of very high slenderness ratios if forced at frequencies that result in low spatial wave numbers. However, as the wave number increases the rotatory inertia and shear deformation become more pronounced and should be included in the solution.

Equation (1) represents Hamilton's extended principle for the system shown in Fig. 1 (Meirovitch, 1967). In Eq. (1) $m$ represents the mass per unit length and $E$ and $I$ represent the modulus of elasticity and the moment of inertia, respectively. $y, \dot{y}$, and $\ddot{y}$ represent the transverse displacement, velocity, and acceleration, respectively, $K_{\mathrm{p}}, K_{\mathrm{c}}$, $C_{\mathrm{p}}$, and $C_{\mathrm{c}}$ are, respectively, the stiffness coefficients and viscous damping coefficients of the foam pad and bungee cord. $\delta$ is the variation symbol, and primes indicate spatial derivatives.

$$
\int_{t 1}^{t 2}\left(\begin{array}{c}
-\int_{0}^{L}\left(m \ddot{y}-E I y^{(4)}\right) \delta y d x-\left.E I y^{\prime \prime} \delta y^{\prime}\right|_{0} ^{L}+\left.E I y^{\prime \prime \prime} \delta y\right|_{0} ^{L}-\left.K_{\mathrm{p}} y \delta y\right|_{x=0} \\
-\left.K_{\mathrm{c}} y \delta y\right|_{x=L}+\left.C_{\mathrm{p}} y \delta y\right|_{x=0}+\left.C_{\mathrm{c}} y \delta y\right|_{x=L}
\end{array}\right) d t=0 .
$$

Stationarity of the system requires that the integrand of Eq. (1) must equal zero. Providing this result leads to the fourth-order Bernoulli-Euler beam equation and the appropriate boundary conditions. Solving this fourth-order homogeneous partial differential equation provides the spatial component of the beam displacement as a function of position and frequency as shown by Eq. (2).

$$
\begin{aligned}
y(x, \omega)= & C_{1} \exp (-i k x)+C_{2} \exp (i k x) \\
& +C_{3} \exp (-k x)+C_{4} \exp (k x) .
\end{aligned}
$$

In Eq. (2), $i$ represents the square root of -1 , $k$ is the complex wave number, and $x$ is the spatial coordinate along the length of the beam. The coefficients $C_{1}, C_{2}, C_{3}$, and $C_{4}$ are determined from the boundary conditions derived from Hamilton's extended principle as shown by the last five terms in Eq. (1).

In this analytical approach, a point force can be added to the system at any position along the structure by imposing continuity of displacement, slope, moment, and a force balance at the location of the applied point force. Due to the area over which the force acts, a point force is not an exact representation of an experimentally applied force. It will be shown that the effect of the point force is manifested as a discontinuity in the analytical power flow results. Given the expression for the displacement field, and using prescribed boundary conditions, the power-flow equations for the analytical model can be developed.

\section{Power Flow in Beams}

Power is defined as the time-averaged product of a generalized force with the in-phase component of a generalized velocity in the direction of the force. When complex numbers are used in the time-harmonic analyses, the power calculation is obtained by taking the dot product of two complex vectors (i.e., the generalized force and velocity). The dot product of two complex vectors is defined as taking the real part of the first vector multiplied by the complex conjugate of the second vector. This process can also be thought of as multiplying one complex number by the in-phase part of another complex number, as discussed by Hambric (1990b), and as shown by Eq. (3), where \langle\rangle indicate the time average, and * implies the complex conjugate.

power

$$
\begin{aligned}
& =\langle\text { generalized force, } \text { generalized velocity }\rangle \\
& =\frac{1}{2} \operatorname{Re}[\text { force velocity*] }
\end{aligned}
$$

In the literature, power or energy flow in a structure is commonly referred to as power flow. The term power flow is derived from the fact that because the direction of the generalized forces are known, the direction of the power is also known. Hence the power can be represented as a vector because both the magnitude and direction are known. When plotting power vectors a 
flow-type pattern is generated, hence the term power flow.

Real or active power flow as discussed by Gavric and colleagues (1990), represents the net energy flow in the structure. Locations of energy sources and sinks can be identified from plots of the active power flow. Imaginary or reactive power flow does not contribute to the net energy flow in the structure as discussed by Pascal et al. (1994). Only active power flow results will be discussed here, even though the procedure presented can easily develop a spatial representation of the reactive power flow.

Because beams are 1-D, power flow only travels in the plus or minus direction along the length of the beam. Analytical and experimental power flow methods that account for 2-D flow in plates and shells were developed previously (Noiseux, 1970; Pavic, 1976). Blotter and West (1995) presented a SLDV approach that builds on the approach presented here and computed the power flow in plates using fifth-order B-spline surface elements to represent the 3-D velocity field measured by the SLDV.

Under the assumed Bernoulli-Euler beam model there are two generalized forces and associated generalized velocities that contribute to the power flow in beams when only transverse motion is considered. The two generalized forces are the shear force and the bending moment. The associated generalized velocities are, respectively, the transverse velocity and the angular velocity. Expressions for the shear and bending moment active power-flow components in terms of the complex-valued transverse displacement, $y$, are shown by Eqs. (4) and (5), respectively. The reactive power-flow component is obtained by taking the imaginary part of Eqs. (4) and (5) instead of the real part.

$$
\begin{gathered}
q_{s}=\frac{1}{2} \operatorname{Re}\left[\left(E I \frac{\partial^{3} y}{\partial x^{3}}\right)\left(\frac{\partial y}{\partial t}\right)^{*}\right], \\
q_{m}=\frac{1}{2} \operatorname{Re}\left[\left(E I \frac{\partial^{2} y}{\partial x^{2}}\right)\left(-\frac{\partial y}{\partial t \partial x}\right)^{*}\right] .
\end{gathered}
$$

\section{Material Properties}

The material properties of a beam that effect the power flow are the structural damping factor, $\eta$, the modulus of elasticity, $E$, and the density, $\rho$. Although known ranges for these values exist, slight variation in these material properties, especially the structural damping factor, has a large effect on the power-flow results and can completely change the analytically predicted energy path. It is not the intent of this study to determine the exact values for the material properties of the test structure. The intent is to show the sensitivity of the power flow, given assumed material properties in the analytical model.

The structural damping factor is the first material property to be discussed. If the structure has zero damping, there is no spatial energy transfer or power flow. As damping is increased, the shear forces and bending moments that develop provide a mechanism for energy transport. Therefore, increasing the structural damping increases the generalized forces, which in turn causes an increase in the power flow. Damping enters the analytical solution through the complex wave number, $k$, and the complex modulus of elasticity, $E_{\mathrm{c}}$, shown by Eqs. (6) and (7).

$$
\begin{gathered}
k^{4}=\omega^{2} \frac{\rho s}{E I} \frac{(1-i \eta)}{\left(1+\eta^{2}\right)}, \\
E_{\mathrm{c}}=E(1+i \eta) .
\end{gathered}
$$

The effects of changes in the structural damping factor are best shown by presenting powerflow results. Figures 2 and 3 show the analytical power-flow results of the same test structure where the only change is an increase from 0.001 to $\mathbf{0 . 0 0 5}$ in the structural damping factor.

In Figs. 2 and 3 a free hanging beam was used as the test structure. The beam was a $139.0 \times 7.6$ $\times 0.7 \mathrm{~cm}, 304$ stainless steel beam. The modulus

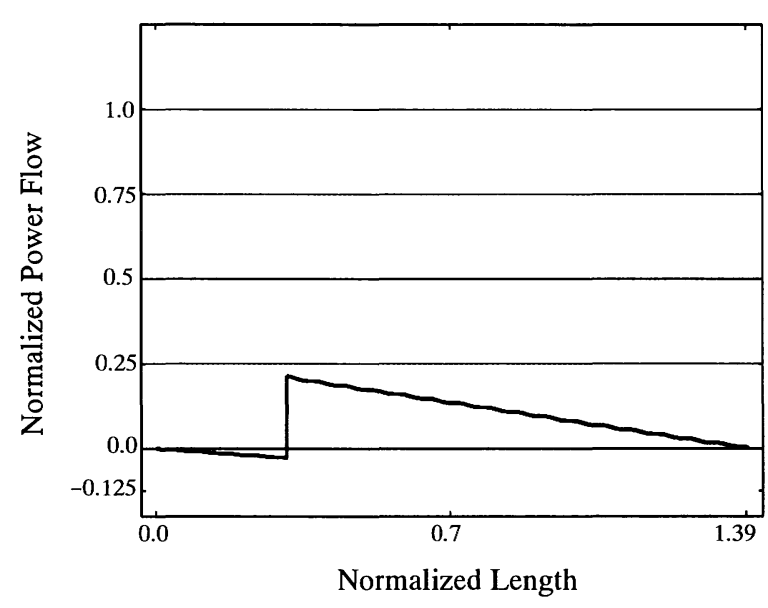

FIGURE 2 Power flow damping 0.001 . 


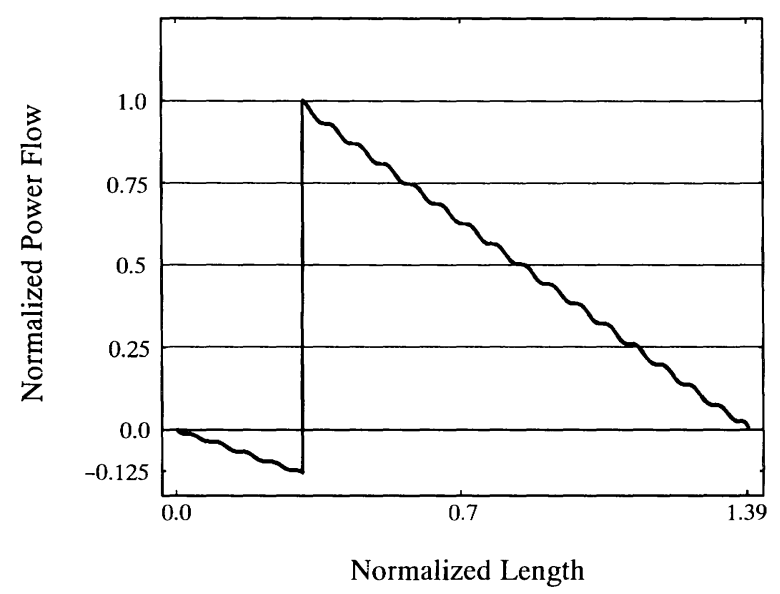

FIGURE 3 Power flow damping 0.005 .

of elasticity, density, and Poisson's ratio were, respectively, $204 \mathrm{GPa}, 7860 \mathrm{~kg} / \mathrm{m}^{3}$, and 0.29 . These parameters were the same parameters of the beam used in the simulated experimental process. The beam was forced at the quarter span with a frequency of $3250.0 \mathrm{~Hz}$. Figures 2 and 3 show that the normalized power flow with a structural damping factor of 0.001 is approximately $20 \%$ of the power flow of the same sysiem with a structural damping factor of 0.005 . The power flow over the structure was normalized between -1 and 1 by dividing by the maximum absolute value of the power flow in the model with structural damping of 0.005 . This implies that an error in the estimation of the structural damping factor of $20 \%$ results in an error in the power flow of approximately $20 \%$ for this system. This is significant because estimation of the damping factor is a difficult task and significant error can result. It should not be presumed that power flow is linearly proportional to the structural damping factor.

The other material properties that effect the power flow are the modulus of elasticity and the density. A change in the modulus of elasticity results in a one to one change in the power flow as shown by Eqs. (4) and (5). The density enters the solution through the wave number as shown by Eq. (6). It acts similarly to the structural damping (i.e., as the density increases the power flow also increases). The modulus of elasticity and the density can have a significant impact on the power flow. However, the estimates of these two material properties are usually known with considerably more certainty than the structural damping factor.

\section{Boundary Conditions}

The exact boundary conditions of any structure are at best difficult to estimate. For example, a beam hanging from a bungee cord or fishing line is often modeled as a free-free beam. However, during testing, the participation of the suspension system can be visually detected even when much care is taken in the setup. Many attempts are made to analytically model various boundary conditions by adding springs and dampers. For example, a free hanging beam would have springs and dampers modeling the suspension. Although springs and dampers can theoretically improve the model, it is still a difficult task to know the appropriate stiffness and damping coefficients of these components, or if another boundary condition model would be a better approximation.

To illustrate the effects of added springs and dampers to a system, power-flow calculations were made on two models of the same test system. The system modeled was a beam hanging from a bungee cord as shown in Fig. 1. The material properties were the same as those listed in the previous section. The first model treated the beam as a true free-free beam. The second model accounted for the suspension system and the sway pad by adding springs and dampers at the beam ends. The spring constant for both springs was $17.5 \mathrm{~N} / \mathrm{m}$ and the viscous damping coefficient was $0.2 \mathrm{~N} \mathrm{~s} / \mathrm{m}$. In both models, the beam was forced with the same magnitude and frequency at the midspan in the transverse direction.

Figure 4 presents the analytically determined power-flow results for the two models. The re-

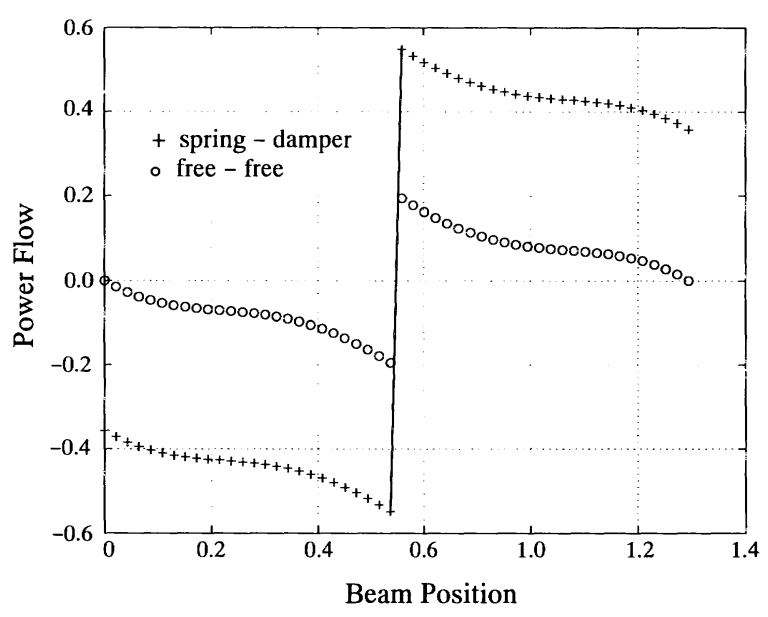

FIGURE 4 Effects of boundary conditions on power flow. 
sults show that for the true free-free beam the power flow was zero at the free ends and maximum at the midspan where the force was applied. This implies that all the energy put into the system by the force was being dissipated along the beam (i.e., no energy is leaving through the free ends of the beam). The case where the bungee cord suspension was modeled with springs and dampers shows that the overall power flow decreases slightly along the length of the beam but a significant amount of energy is absorbed by the boundary. It is also illustrated that even though the magnitude of the force was the same in both cases, due to the differences in the system, more energy was put into the beam with the applied spring-damper boundary conditions.

\section{Near-Field Effects}

Near-field effects arise due to points of discontinuity in the system such as locations of the force and boundaries. If the structural damping factor is small, many investigators neglect the near-field effects. By neglecting the near-field effects, the four-term beam displacement given by Eq. (2) reduces to a two-term equation. This greatly simplifies the analytical solution. However, it will be shown that the near-field effects are significant and should be accounted for in the solution, especially at low frequencies.

To illustrate the near-field effects, the kinetic energy for a free-free beam was calculated using two models. The first model accounted for the near-field effects and the second model neglected their contribution. Figure 5 shows the normalized results of the two models for the first half of the beam. The near-field effects of the force at position 12 and the free boundary at position 0 are shown. Without near-field effects in the solution, the peaks of the kinetic energy are shown to be constant. However, when near-field effects are included it is shown that both the magnitude and the spatial phase of the kinetic energy change. In beams when the near-field effects are neglected, the kinetic and potential energy are equal in magnitude, spatially in phase (reach maximum and minimum at the same spatial location), and $180^{\circ}$ out of phase in time. When near-field effects are present none of these statements are necessarily true.

Figure 5 also indicates that it takes about one and a half wavelengths for the near-field effects to fade away. This implies that for low frequencies, near the first few modes, significant near-field effects can be present throughout the entire structure, even if the structural damping coefficient is small.

\section{EXPERIMENTAL APPROACH}

An overview of the three-step ESPF approach is presented in this section. The development of this three-step approach is the main contribution of this research. The novelty of this approach with

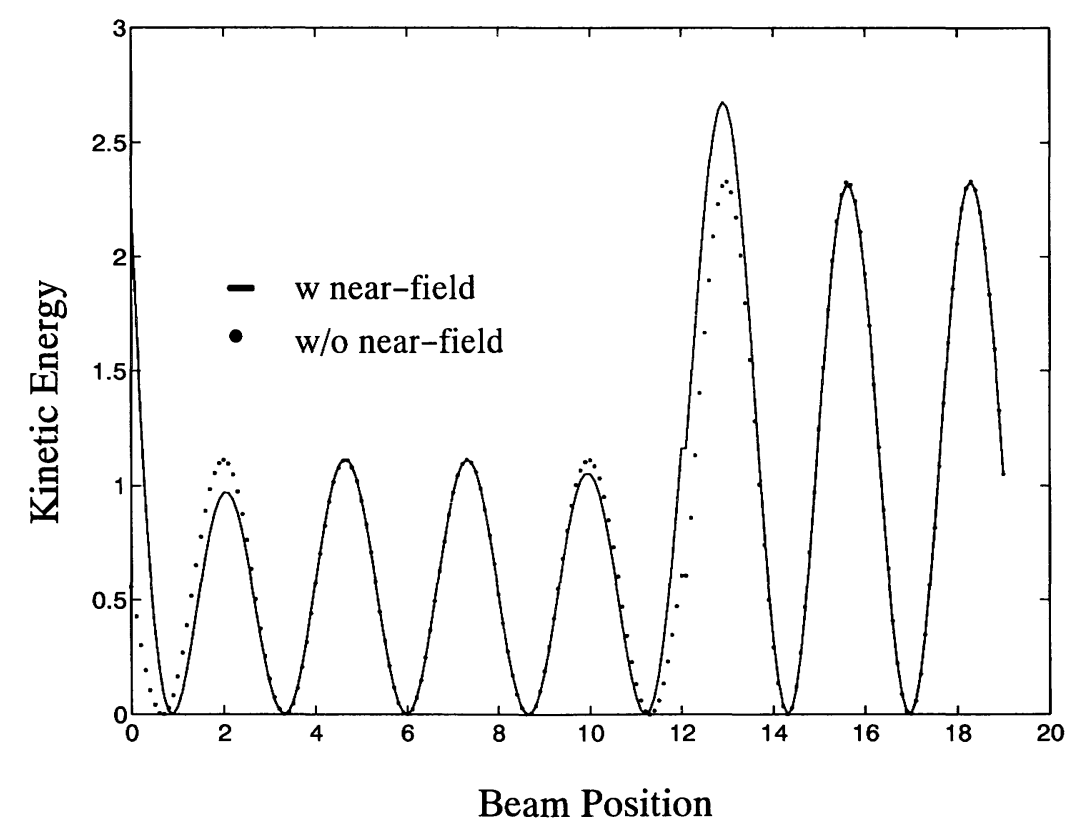

FIGURE 5 Kinetic energy with and without near-field effects. 
respect to current literature will be discussed throughout the remainder of the article.

The first step of the ESPF approach consists of measuring the dynamic response of the structure with an SLDV. The SLDV measures the response of the vibrating structure that inherently contains the true damping mechanisms, boundary conditions, and near-field effects, and provides the data to solve for a spatial model of the system response. The SLDV contain a 1-mW helium-neon laser of wavelength $632.8 \mathrm{~nm}$. The SLDV functions as a noncontacting velocity transducer capable of remote measurement of the velocity of a solid surface. Velocities in the range of $0- \pm 1000$ $\mathrm{mm} / \mathrm{s}$ can be measured at working distances up to $200 \mathrm{~m}$. The SLDV incorporates scanning mirrors to permit controlled deflection of the laser beam and therefore the measurement position across the structure. The SLDV is based on a Michelson interferometer in which a laser beam is divided into reference and signal beams. The signal beam is directed onto a vibrating test surface that changes the frequency of the back-reflected light. This reflected signal beam is recombined with the internal reference beam where the Doppler shift between the two beams is directly proportional to the measured velocity. The primary instrument output is an analog voltage proportional to surface velocity.

The second step of the ESPF process consists of solving for the continuous 3-D complex-valued velocity field from the laser data. This is done by using the experimental spatial dynamics modeling (ESDM) technique as discussed by Montgomery (1994). It is emphasized that raw 2-D images of the velocity field are not used directly to compute the power flow.

In the third step of the ESPF process, high order B splines are used to represent the velocity field. This allows a continuous representation of the spatial and temporal derivatives of the velocity field that are required to compute the power flow. In most cases, a minimum of fifth-order Bspline elements that allow quadratic representation of the third derivative should be used. The total experimental power flow can then be computed spatially in the structure as shown by Eqs. (4) and (5).

\section{EXPERIMENTAL AND ANALYTICAL RESULTS}

This section presents the results of the experimental power-flow process described in the previ- ous sections. In each step of the process, results from two models of the same test structure are presented. The first model was a simulated experimental model where the dynamic response was generated by a computer simulation of the SLDV. This computer simulation accounts for noise on the velocity signal and for typical experimental error in the process. The second model was the analytical process in which the response and boundary conditions were obtained from Hamilton's extended principle as shown by Eq. (1). This model represents the ideal case in which zero noise or experimental error are present. The differences between the experimental model and the analytical model are due to noise on the velocity signal from the SLDV and experimental error, such as errors in the SLDV registration process, calibration factors, and signal processing. Because the focus of this work was to develop an experimental approach to power flow and not to determine the correct material properties and spring/damper coefficients of the system, reasonable approximations were obtained for these parameters by a simple manual system identification process.

The system under test in each case was a 139.0 $\times 7.6 \times 0.7 \mathrm{~cm}, 304$ stainless steel beam hanging from a bungee cord/fishing line suspension as shown in Fig. 1. The material properties used in the analytical models were the same as those listed in the Material Properties section. The beam was forced in the transverse direction at the bungee cord end with a frequency of $15 \mathrm{~Hz}$. This frequency was close to the second resonance frequency of the beam/suspension system. It should be noted that if the beam was forced exactly at resonance, only standing waves would be present in the system and the active power flow would be zero as discussed by Gavric et al. (1990).

The dynamic response of the experimental model was obtained as discussed in the previous section. The optimal fit for this response was obtained by using four fifth-order B-spline elements to fit the centerline data. Figures 6 and 7 illustrate the real and imaginary velocity components of the analytical beam model. Figure 8 shows a plot of the residuals between the real velocity computed in the experimental model and the analytical model representations. The residuals had a mean value of $-5.16 \times 10^{-9} \mathrm{~m} / \mathrm{s}$ and the variance was calculated to be $1.65 \times 10^{-9} \mathrm{~m} / \mathrm{s}$. The residuals between the experimental and the analytical imaginary velocity models are shown in Fig. 9. The residuals had a mean value 


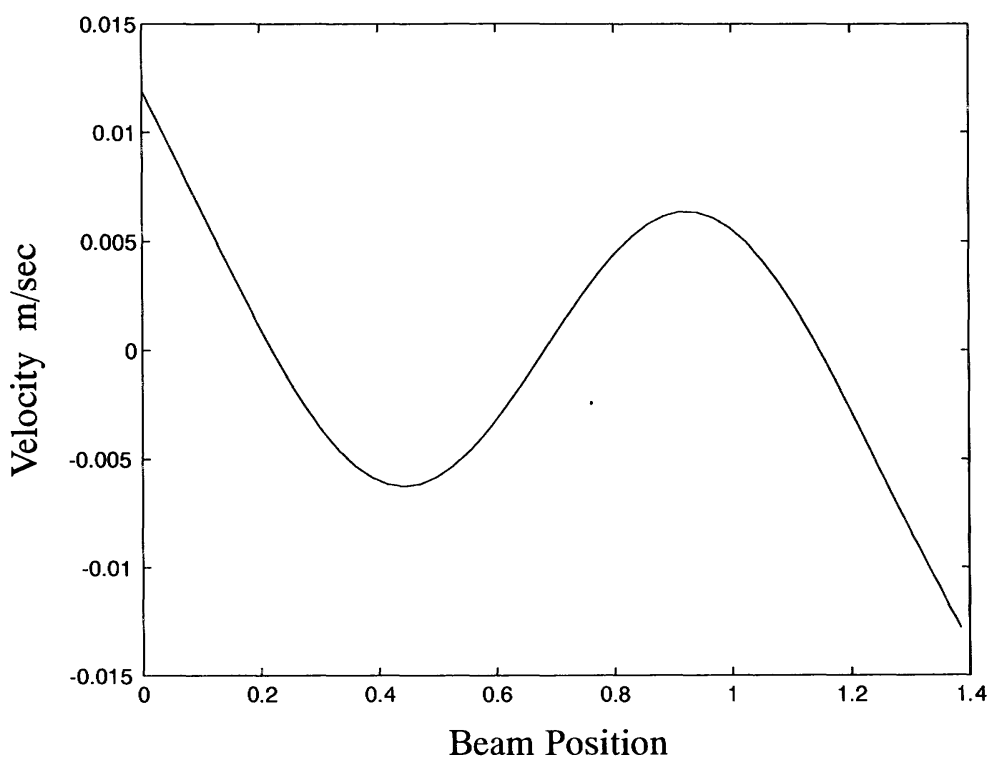

FIGURE 6 Real component of the velocity field over the beam.

of $1.27 \times 10^{-6} \mathrm{~m} / \mathrm{s}$ and a variance of $8.37 \times$ $10^{-7} \mathrm{~m} / \mathrm{s}$.

Once an acceptable B-spline representation of the response is obtained, the power flow can be computed. It is important to follow the powerflow calculation through by examining the effects of taking the required derivatives. Figure 10 shows that even though a tight band on the imaginary velocity residuals was obtained, significant differences in the third derivative computations were generated from the initially small differences between the two representations. It is further illustrated that the error is largest near the ends of the beam. Due to the error that is magnified by computing the third derivative, other methods based on energy instead of displacements were investigated by many researchers (Halkyard and Mace, 1994). The error in this result also implies that future work should concentrate on converging the power flow instead of the displacements. This process would be similar to the methods used in finite element analysis when convergence

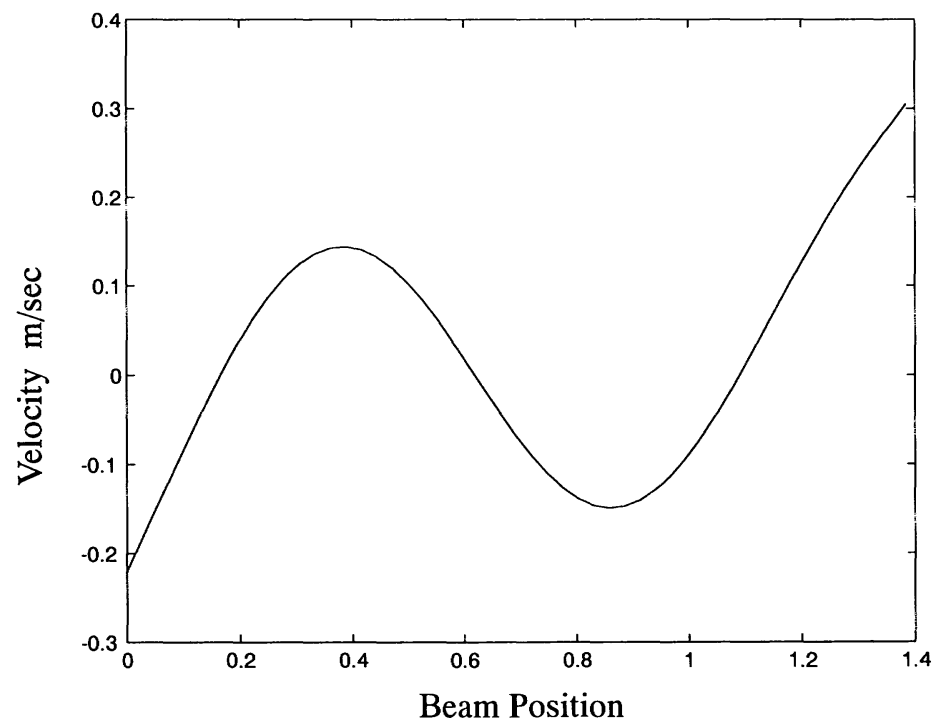

FIGURE 7 Imaginary component of the velocity field over the beam. 


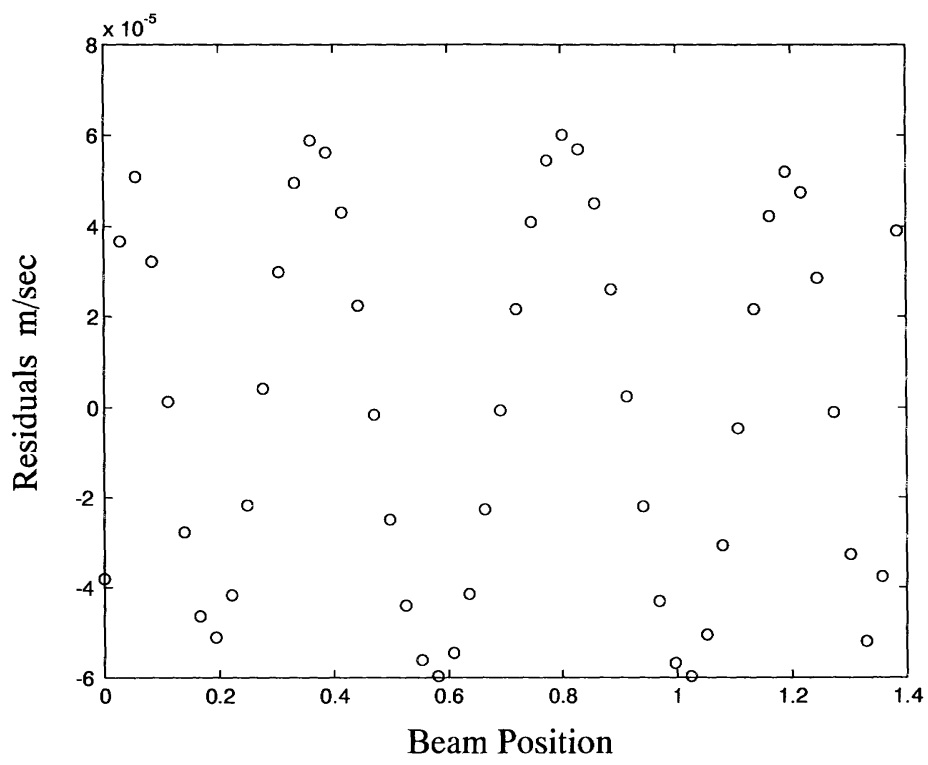

FIGURE 8 Real velocity residuals (experimental vs. analytical).

of the stresses is performed instead of convergence of the displacements. The implication of this result is to converge the quantity of interest that in this case is the power flow and not the velocity.

After computing the required derivatives, the shear and moment components of power flow were computed as shown by Figs. 11 and 12, respectively. It is shown that the power flow due to the bending moment has a much smaller error than the power flow due to the shear force. This is due to the error that is manifested in the third derivative computation as compared to the second derivative computation.

Adding to the results of Figs. 11 and 12 provides the total power flow as shown by Fig. 13. Figure 13 shows that the energy enters the system through the shaker at beam position 1.39 and travels along the beam in a negative direction. Energy dissipation along the length of the beam is due

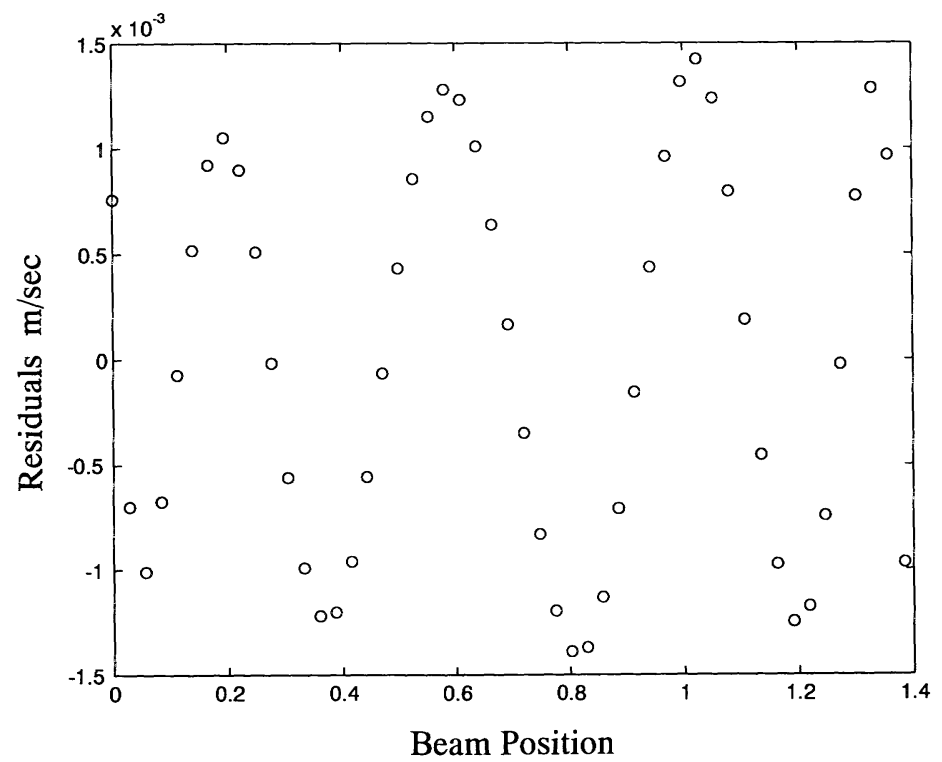

FIGURE 9 Imaginary velocity residuals (experimental vs. analytical). 


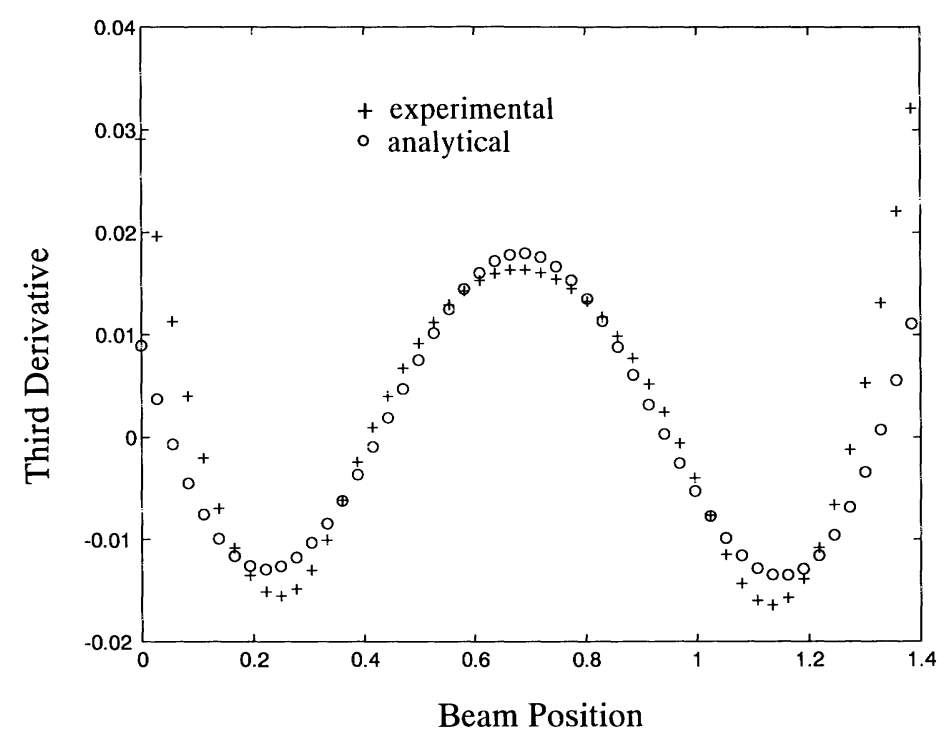

FIGURE 10 Third spatial derivative of the displacement field.

mainly to internal damping of the structure. At beam position 0 the energy dissipated by the spring/damper is shown. The largest differences between the two power-flow models was $14 \%$ and occurs near the boundaries. The differences in the power-flow results between the experimental and the analytical methods are due mainly to experimental error. Although a perfect representation of the power flow is not obtained by the ESPF method, the general flow pattern along with the locations of the sources and sinks can be identi- fied. The ESPF model can be improved by converging the power-flow field instead of the velocity field and by reducing the experimental error in the data acquisition process.

\section{SUMMARY}

A spatially continuous model of the power flow in a system is significant for both dynamic analysis as well as design. The ability of the system to

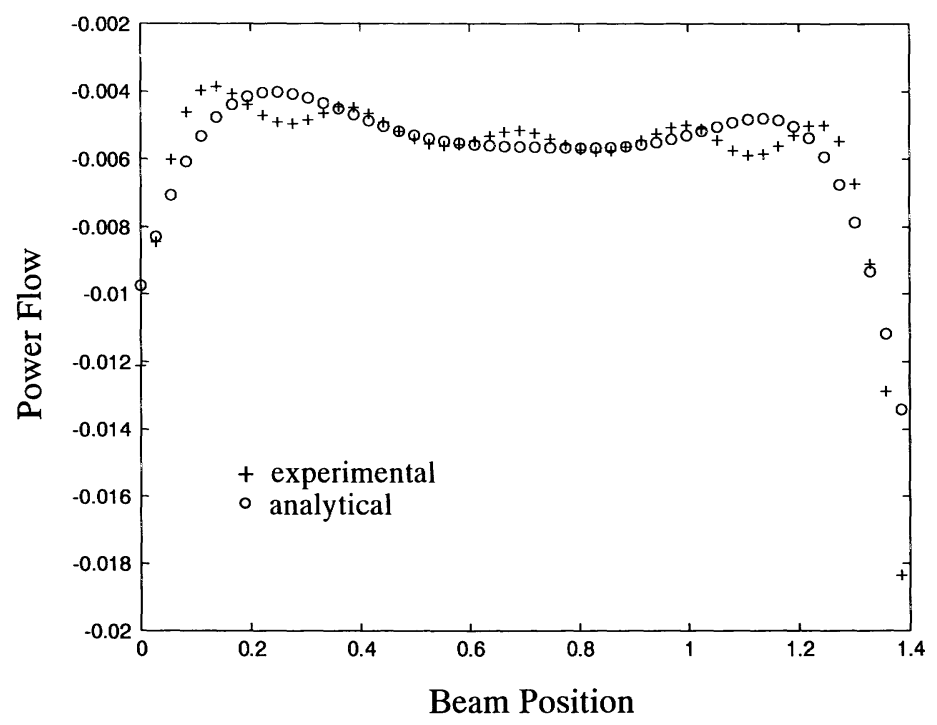

FIGURE 11 Shear power-flow component. 


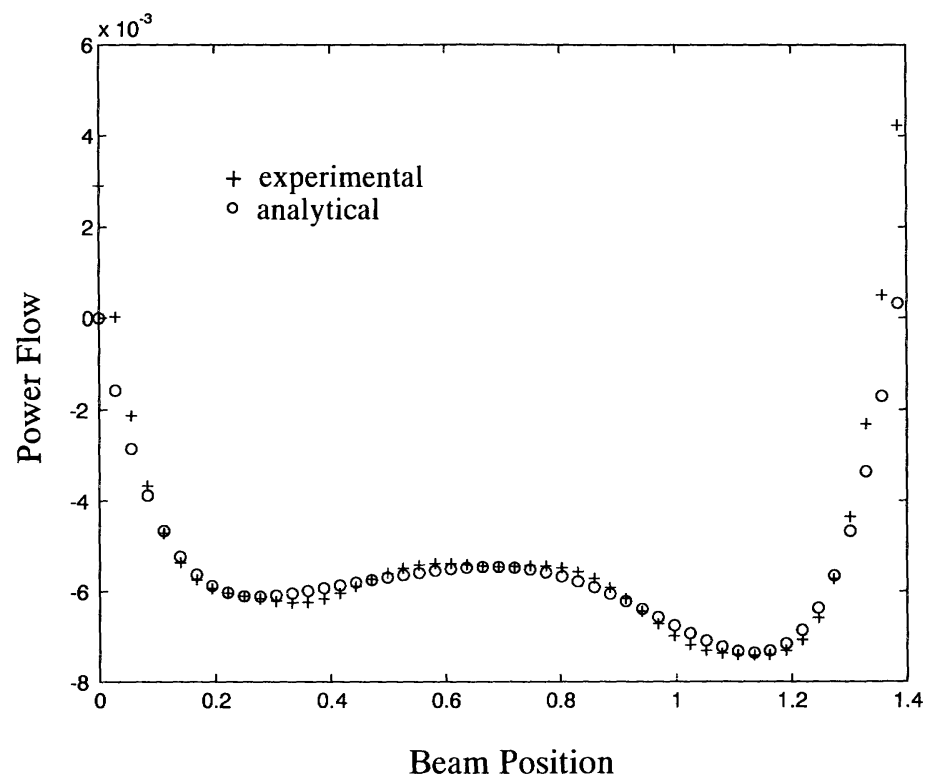

FIGURE 12 Moment power-flow component.

transfer energy as a function of time, space, and frequency is a unique signature obtained from a power-flow analysis that can be used to characterize the system. In a design scenario, power-flow results could be used to design the channeling or dissipation of the energy in the structure.

Many experimental and analytical methods have been developed that attempt to predict the power flow in a structure. This research has shown that to extract the true power flow in a system, a spatial measurement of the structure's dynamic response that includes experimental measurement of the boundary conditions is required. Current experimental power-flow methods are incapable of providing this required spatial representation of the system's dynamics. It was also shown and discussed that even though a spatial representation of the power flow can be obtained from an analytical solution, the underlying assumptions on the material properties and the boundary conditions required to obtain a solution can significantly corrupt the results. The

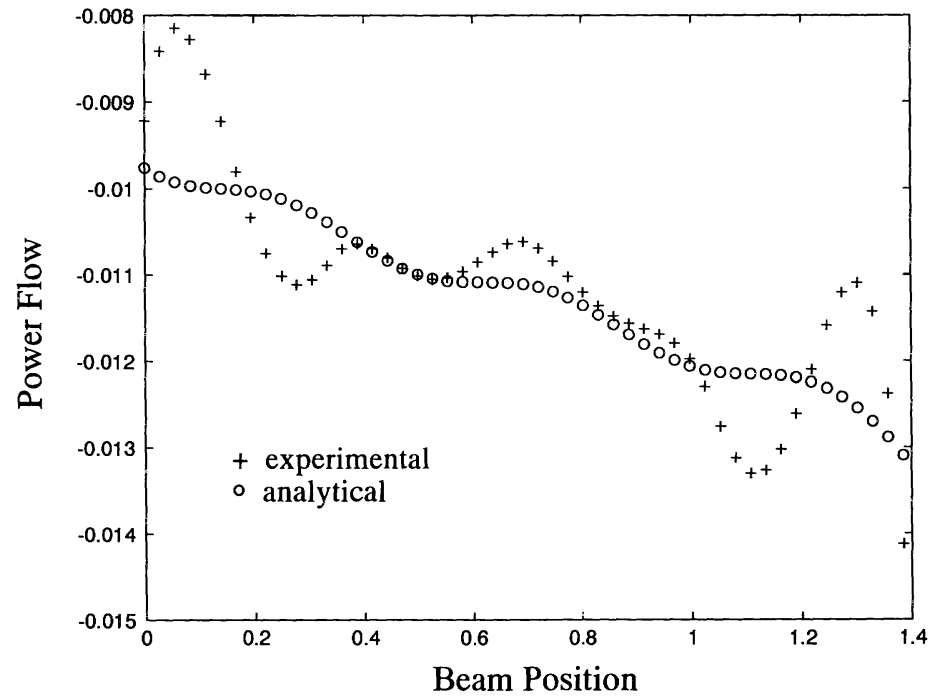

FIGURE 13 Total power flow. 
ESPF method presented in this research is a novel approach because a spatially continuous representation of the power flow that experimentally measures the boundary conditions is provided.

The key concept that sets the ESPF approach apart from other methods is developed around providing a spatial representation of the power flow that satisfies the true physics of the system. The ESPF approach consists of using an SLDV to extract a spatial representation of the system response. From the laser data, a continuous 3-D complex-valued velocity field is generated. From this representation of the system dynamics, a spatially continuous mapping of the power flow can be computed.

The experimental power-flow method developed was presented and validated against analytical results. The validation for the experimental method consisted of comparing the results of a prescribed analytical model with known material properties and boundary conditions to a simulated experimental model that accounted for signal noise and experimental error. The experimental results were shown to compare within $14 \%$ of the analytical method throughout the domain. The impact of structural damping, boundary conditions, near-field effects, and the effects of taking the third spatial derivative were quantified and discussed.

Future applications of this method will be to perform acoustic prediction, adaptive control, health monitoring, damage detection, and design for dynamics of vibrating structures. The method will also be used to extract system parameters within a system identification framework to update analytical/computational models.

\section{REFERENCES}

Blotter, J. D., and West, R. L., "'Power Flow in Plates Using a Scanning Laser Doppler Vibrometer," in 36th AIAA/ASME/ASCE/AHA/ASC Structures,
Structural Dynamics, and Materials Conf., 1995, Part 3, pp. 1835-1843.

Bouthier, O. M., and Bernhard, R. J., 1992, "Models of Space-Averaged Energetics of Plates," AIAA Journal, Vol. 30, pp. 616-623.

Gavric, L., Carniel, X., and Pavic, G., "StructureBorne Intensity Fields in Plates, Beams and PlateBeam Assemblies," in 3rd International Congress on Intensity Techniques, 1990, Senlis, France, pp. 223-230.

Halkyard, C. R., and Mace, B. R., "A Wave Component Approach to Structural Intensity in Beams," in 4th International Congress on Intensity Techniques, 1994, Senlis, France, pp. 183-190.

Hambric, S. A., "Influence of Different Wave Motion Types on Finite Element Power Flow Calculations," in 3rd International Congress on Intensity Techniques, 1990a, Senlis, France, pp. 215-222.

Hambric, S. A., 1990b, "Power Flow and Mechanical Intensity Calculations in Structural Finite Element Analysis," Journal of Vibrations and Acoustics, Vol. 112, pp. 542-549.

Meirovitch, L., 1967, Analytical Methods in Vibrations, Macmillan, New York, p. 44.

Montgomery, D. E., "Three Dimensional Experimental Spatial Dynamic Response of a Reciprocating Freon Compressor," Journal of Optics and Lasers in Engineering, to appear.

Noiseux, D. U., 1970, "Measurement of Power Flow in Uniform Beams and Plates," Journal of the Acoustical Society of America, Vol. 47, pp. 235-247.

Palmer, J. D., Williams, E. J., and Fox, C. H. J., "Energy Flow Analysis in Built-Up Structures," in 3rd International Congress on Intensity Techniques, 1993, Senlis, France, pp. 313-322.

Pascal, J. C., Carniel, X., Chalvidan, V., and Smigielski, P., "Determination of Phase and Magnitude Maps for Vibration Flow Measurements in Structures Using Interferometric Cineholography," in First International Conference on Vibration Measurements by Laser Techniques: Advances and Applications, 1994, Ancona, Italy, pp. 555-566.

Pavic, G., 1976, "Measurement of Structure-Borne Wave Intensity Part 1: Formulation of the Method,' Journal of Sound and Vibration, Vol. 49, pp. 221-230. 

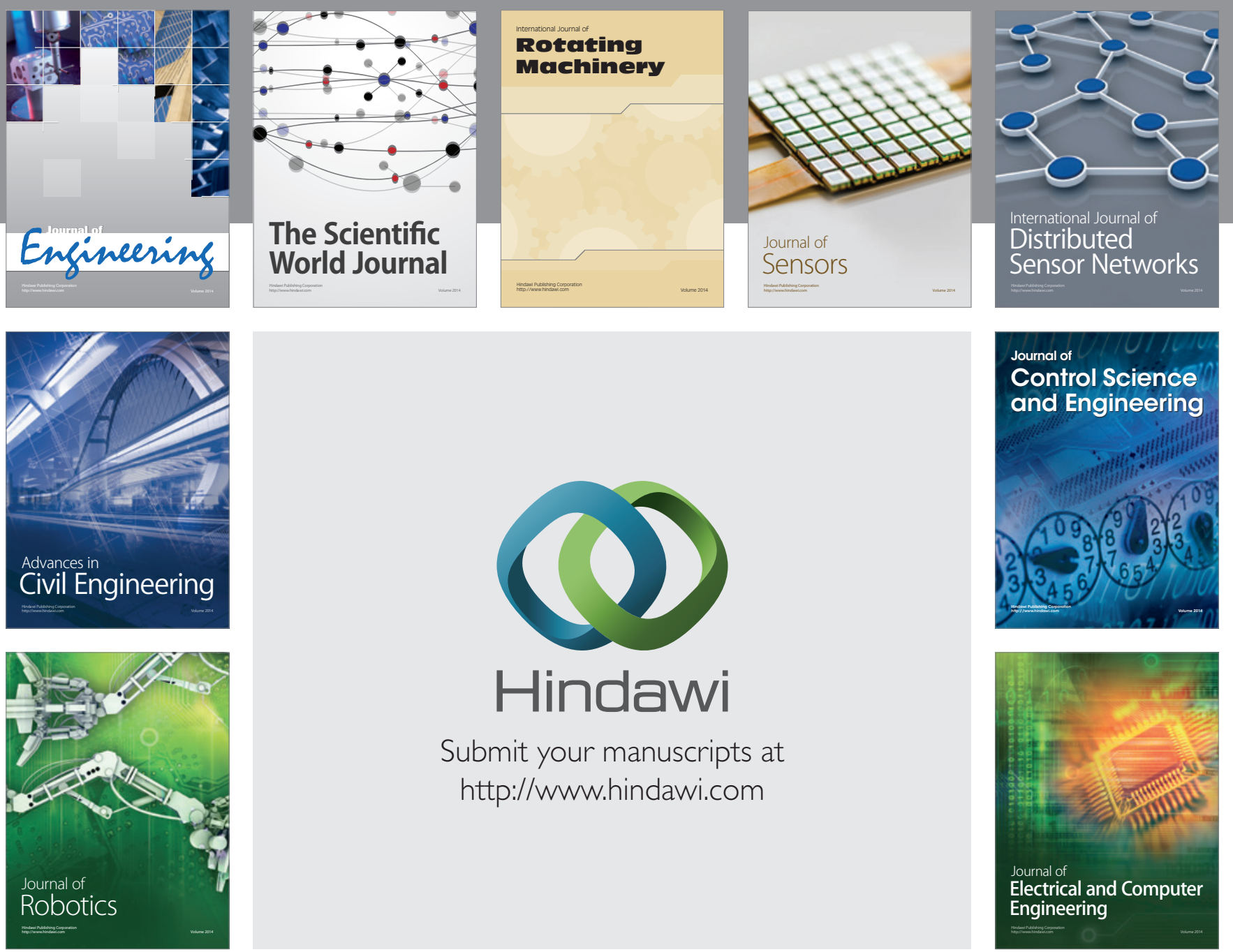

Submit your manuscripts at

http://www.hindawi.com
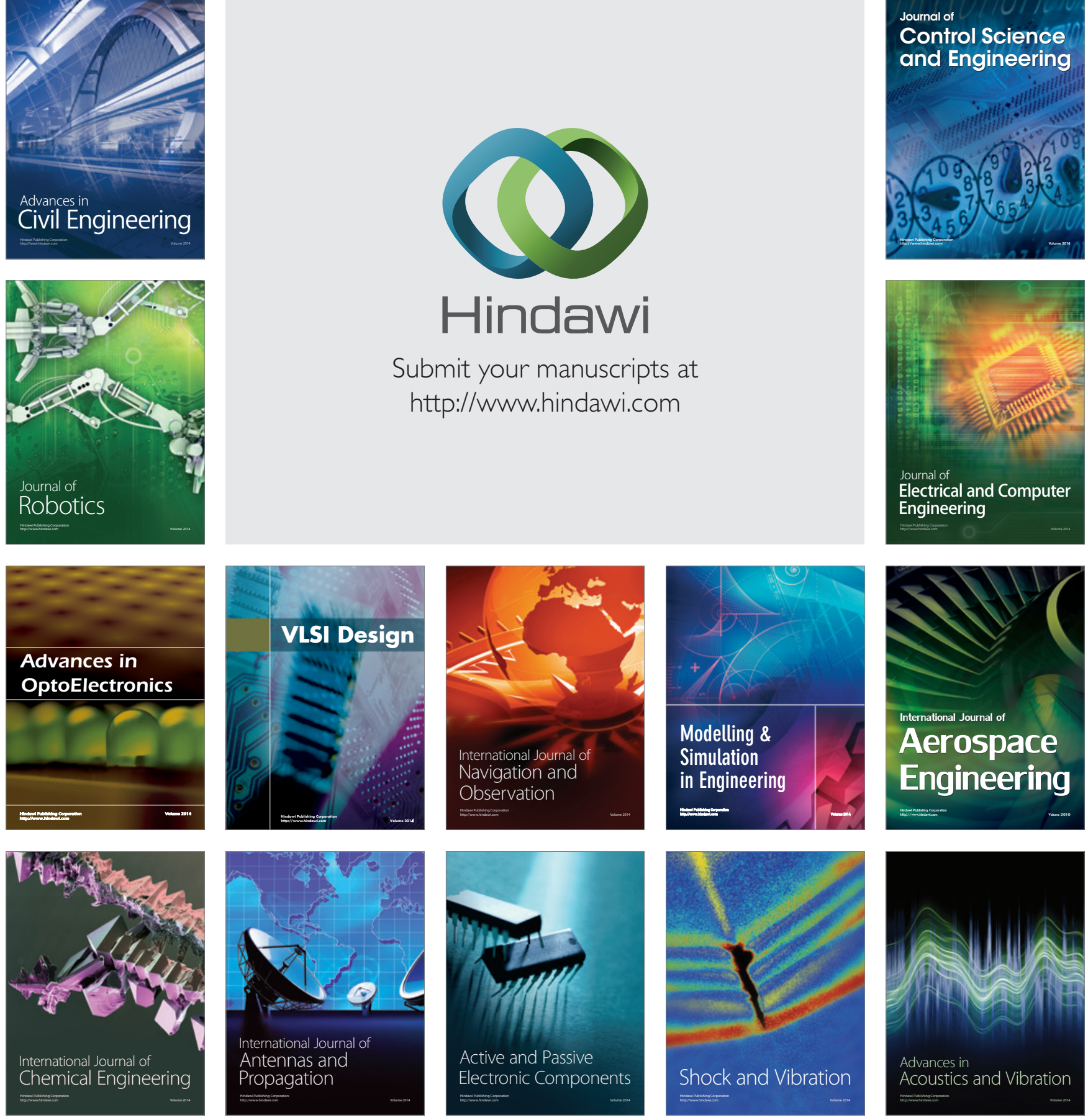